\title{
Linarin enhances melanogenesis in B16F10 cells via MAPK and PI3K/ AKT signaling pathways
}

\author{
So-Yeon $\mathrm{Oh}^{1} \cdot$ Jin Kyu Kang ${ }^{1} \cdot$ Chang-Gu Hyun ${ }^{1}$
}

Received: 24 October 2021 / Accepted: 16 November 2021 / Published Online: 31 December 2021

(C) The Korean Society for Applied Biological Chemistry 2021

\begin{abstract}
In this study, we discovered for the first time that linarin, a flavonoid compound, enhances melanin biosynthesis in B16F10 cells, and subsequently elucidated the underlying mechanism of linarin-induced melanogenesis. Linarin showed no cytotoxicity at a concentration of $42 \mu \mathrm{M}$ and significantly increased intracellular tyrosinase activity and melanin content in B16F10 cells. Mechanistic analysis showed that linarin increased the expression of tyrosinase, tyrosinase-related protein 1 (TRP-1), and microphthalmia-associated transcription factor (MITF) that are related to melanogenesis. Moreover, linarin decreased the phosphorylation of extracellular signal-regulated kinase (ERK) and protein kinase B (AKT). Finally, we evaluated the effect of the structure-activity relationship of linarin and its aglycone on melanogenesis. The results indicated that linarin enhances the expression of melanogenic proteins by activating MITF expression via the modulation of mitogen-activated protein kinase (MAPK), phosphatidylinositol 3-kinase (PI3K), and protein kinase B signaling pathways in B16F10 cells, thereby enhancing melanogenesis.
\end{abstract}

Keywords B16F10 cells · Linarin - Melanogenesis - Mitogenactivated protein kinases $\cdot$ Phosphatidylinositol 3-kinase/protein kinase B

Chang-Gu Hyun $(\bowtie)$

E-mail: cghyun@jejunu.ac.kr

${ }^{1}$ Jeju Inside Agency and Cosmetic Science Center, Department of Chemistry and Cosmetics, Jeju National University, Jeju 63243, Republic of Korea

This is an Open Access article distributed under the terms of the Creative Commons Attribution Non-Commercial License (http://creativecommons. org/licenses/by-nc/3.0/) which permits unrestricted non-commercial use, distribution, and reproduction in any medium, provided the original work is properly cited.

\section{Introduction}

Melanin is a skin pigment that contributes to human skin color, protects the skin from UV damage, and helps maintain the normal body temperature. Melanogenesis refers to the production of melanin by melanocytes. The absence of melanocytes or loss of pigmentation causes skin diseases such as vitiligo and albinism [1]. Currently, the most preferred treatments to relieve skin damages are skin grafts, lasers, vitamin D analogs, and steroids. However, most of these treatments have various limitations and side effects such as allergies, contact dermatitis, eczema, and cytotoxicity [2]. Therefore, elucidating the underlying mechanisms of melanin pigmentation is necessary to develop new and safe melanogenesis-stimulating products [3]. Linarin is a well-known flavonoid compound exhibiting osteogenic, sedative, sleep-enhancing, antipyretic, anti-inflammatory, neuroprotective, and anticonvulsant properties [4-7]. However, the effects of linarin on melanogenesis have not been evaluated. Here, we report the novel effect of linarin on melanogenesis and signal transduction pathways involved in melanogenesis in murine B16F10 melanoma cells.

\section{Materials and Methods}

\section{Chemicals and reagents}

The linarin used in this study was purchased from Extrasynthese (Genay CEDEX, France). Dulbecco's modified Eagle's medium (DMEM), penicillin/streptomycin, trypsin-ethylenediaminetetraacetic acid, and bicinchoninic acid assay (BCA) protein kit were purchased from Thermo Fisher Scientific (Waltham, MA, USA). Fetal bovine serum (FBS) was purchased from Merck Millipore (Burlington, MA, USA). $\alpha-\mathrm{MSH}$, protease inhibitor cocktail, sodium hydroxide $(\mathrm{NaOH}), \mathrm{L}-\mathrm{DOPA}$, sodium phosphate monobasic, and sodium phosphate dibasic were purchased from SigmaAldrich (St. Louis, MO, USA). Tyrosinase, tyrosinase-related protein (TRP)-1, TRP-2, MITF, and $\beta$-actin primary antibodies were purchased from Santa Cruz Biotechnology (Dallas, TX, 
USA). Protease/phosphatase inhibitor cocktail and extracellular signal-regulated kinase (ERK), AKT, p-AKT, p-ERK primary anti-bodies, anti-mouse secondary antibodies, and anti-rabbit secondary antibodies were purchased from Cell Signaling Technology (Danvers, MA, USA). $2 \times$ Laemmli sample buffer and Tween 20 were purchased from Bio-Rad (Hercules, CA, USA). Skim milk was purchased from BD Difco (Sparks, MD, USA). Bovine serum albumin (BSA) was purchased from Bovostar (Bovogen, Melbourne, Australia). 3-(4,5-dimethylthiazol-2-yl)2,5-diphenyltetrazolium bromide (MTT), dimethyl sulfoxide (DMSO), sodium dodecyl sulfate (SDS), tris-buffered saline (TBS), and enhanced chemiluminescence (ECL) kits were purchased from Biosesang (Sungnam, Gyeonggi-do, Korea).

\section{Cell culture}

B16F10 murine melanoma cells were obtained from ATCC (Manassas, VA, USA). Cells were cultured in DMEM containing $10 \%(\mathrm{v} / \mathrm{v})$ FBS and $1 \%(\mathrm{v} / \mathrm{v})$ penicillin-streptomycin in a humidified incubator at $37{ }^{\circ} \mathrm{C}$ and $5 \% \mathrm{CO}_{2}$. The cells were passaged every three days.

\section{Measurement of cell viability}

The MTT assay was performed to determine the effect of chrysoeriol on cell viability. Cells were seeded into a 24 -well plate at $8 \times 10^{3}$ cells/well for $24 \mathrm{~h}$, treated with $5.3,10.5,21,42$, or 84 $\mu \mathrm{M}$ chrysoeriol and $\alpha-\mathrm{MSH}(100 \mathrm{nM})$, and incubated for $72 \mathrm{~h}$. The supernatant was re-moved, and MTT reagent $(0.2 \mathrm{mg} / \mathrm{mL}$ in DMEM) was added for $4 \mathrm{~h}$. After the reaction time, all MTT reagents and DMSO were removed. Absorbance was measured at $560 \mathrm{~nm}$ using a microplate reader (Tecan, Grödig, Austria).

\section{Measurement of melanin}

The release of melanin from the culture was determined as previously described with modifications [7]. B16F10 cells were seeded into a 6 -well plate at $5 \times 10^{4}$ cells/well for $24 \mathrm{~h}$, treated with $5.3,10.5,21$, or $42 \mu \mathrm{M}$ linarin and $\alpha$-MSH $(100 \mathrm{nM})$, and incubated for $72 \mathrm{~h}$. Then, the supernatant was removed, and the cells were washed once with $1 \times$ PBS. RIPA buffer $(200 \mu \mathrm{L})$ was added, followed by lysis at $4{ }^{\circ} \mathrm{C}$ for $20 \mathrm{~min}$. The cells were added to a $1.5-\mathrm{mL}$ e-tube with a cell scraper and centrifuged at 15,000 rpm for $20 \mathrm{~min}$ at $-8{ }^{\circ} \mathrm{C}$. The pellets were then obtained. The pellet was dissolved in $200 \mu \mathrm{L}$ of $1 \mathrm{~N} \mathrm{NaOH}$ with $10 \%$ DMSO at $80{ }^{\circ} \mathrm{C}$ for $10 \mathrm{~min}$. The absorbance was measured at $405 \mathrm{~nm}$ using a microplate reader (Tecan, Grödig, Austria). Protein concentration was quantified using a BCA protein assay kit.

\section{Intracellular tyrosinase assay}

An intracellular tyrosinase activity assay was performed to determine tyrosinase activity in B16F10 cells. B16F10 cells were seeded into a 6 -well plate at a density of $5 \times 10^{4}$ cells/well for $24 \mathrm{~h}$. The cells were treated with the samples at different concentrations and incubated for $72 \mathrm{~h}$. After washing with $1 \times$ PBS, RIPA buffer containing $1 \%$ protease inhibitor cocktail was added. Cells were lysed at $4{ }^{\circ} \mathrm{C}$ for $20 \mathrm{~min}$. The supernatant was separated by centrifugation under the same conditions used for the melanin content assay. Protein concentration was quantified using a BCA protein assay kit. Then, $20 \mu \mathrm{L}$ of protein $(20 \mu \mathrm{g} / \mathrm{mL})$ and $80 \mu \mathrm{L}$ of L-DOPA $(2 \mathrm{mg} / \mathrm{mL})$ were added to a $96-$ well plate and incubated at $37^{\circ} \mathrm{C}$ for $2 \mathrm{~h}$. Absorbance was measured at $490 \mathrm{~nm}$ using a spectrophotometric microplate reader (Tecan, Grödig, Austria).

\section{Western blot analyses}

B16F10 cells were seeded into a 6 -well plate at $5 \times 10^{4}$ cells/well for $24 \mathrm{~h}$ and treated with $5.3,10.5,21$, or $42 \mu \mathrm{M}$ linarin and $\alpha$ MSH (100 nM). The samples were then treated for 4, 24, 48, and $72 \mathrm{~h}$. After washing with cold $1 \times \mathrm{PBS}$, RIPA buffer with $1 \%$ protease inhibitor cocktail or $1 \%$ protease/phosphatase inhibitor cocktail and lysed at $4{ }^{\circ} \mathrm{C}$ for $20 \mathrm{~min}$. After collecting cells in a 1.5-mL e-tube, centrifugation was performed at $15,000 \mathrm{rpm}$ for $30 \mathrm{~min}$ at $-8{ }^{\circ} \mathrm{C}$, and the supernatant was obtained. Protein concentration was quantified using the BCA protein assay kit. The protein was quantified at $20 \mu \mathrm{g} / \mathrm{mL}$, and the loading sample was heated at $100{ }^{\circ} \mathrm{C}$ for $5 \mathrm{~min}$ with the sample buffer prepared; the protein was quantified in a 1:1 ratio. The loading sample was stored at $-20{ }^{\circ} \mathrm{C}$ before loading. Protein samples were separated by SDS-polyacrylamide gel by size. The isolated proteins were transferred from a gel to a polyvinylidene (PVDF) membrane. The membrane was washed with TBS containing $1 \%$ Tween 20 ( $1 \times$ TBS-T), blocked with $5 \%$ skim milk (in $1 \times$ TBS-T) or $5 \%$ BSA (in $1 \times$ TBS-T) for $1 \mathrm{~h}$, and cleaned 6 times with $1 \times$ TBS-T for $10 \mathrm{~min}$. Antibodies were diluted with $1 \%$ skim milk (in $1 \times$ TBS-T, Santa Cruz) or $1 \%$ BSA (in $1 \times$ TBS-T, Cell Signaling) at a 1:1000 ratio. The membrane was incubated with the primary antibody for more than $12 \mathrm{~h}$ at $4{ }^{\circ} \mathrm{C}$. The secondary antibody diluted at $1: 1000$ to $1 \%$ BSA (in $1 \times$ TBS-T) was then incubated at room temperature for more than $2 \mathrm{~h}$ and washed with $1 \times$ TBS$\mathrm{T}$ for $10 \mathrm{~min}$ for 6 times. Finally, the ECL solution was attached to the secondary antibody, and proteins were detected using Chemidoc (WL, Vilber Lourmat, France).

\section{Statistical analysis}

All results are presented as the mean \pm standard deviation of three replicates. Statis-tical significance was expressed as a $p$-value using Student's t-test. ${ }^{*} p<0.05,{ }^{* *} p<0.01$.

\section{Results and Discussion}

To determine the safe concentrations, the cytotoxicity of linarin was evaluated in B16F10 cells using the 3-(4,5-dimethylthiazol-2yl)-2,5-diphenyltetrazolium bromide assay. The cells were treated with different concentrations of linarin ranging from 10.5-84.0 $\mu \mathrm{M}$. As shown in Fig. 1A, no apparent cytotoxicity was observed in B16F10 cells at linarin concentrations of 10.5, 21.0, and 42.0 
(A)

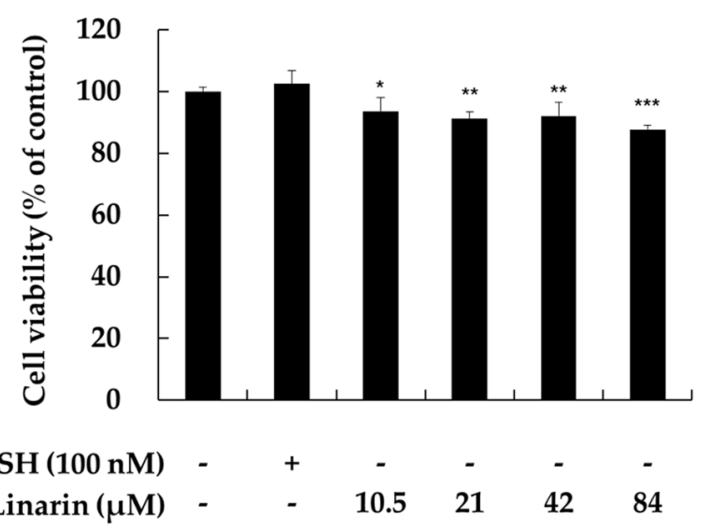

(B)

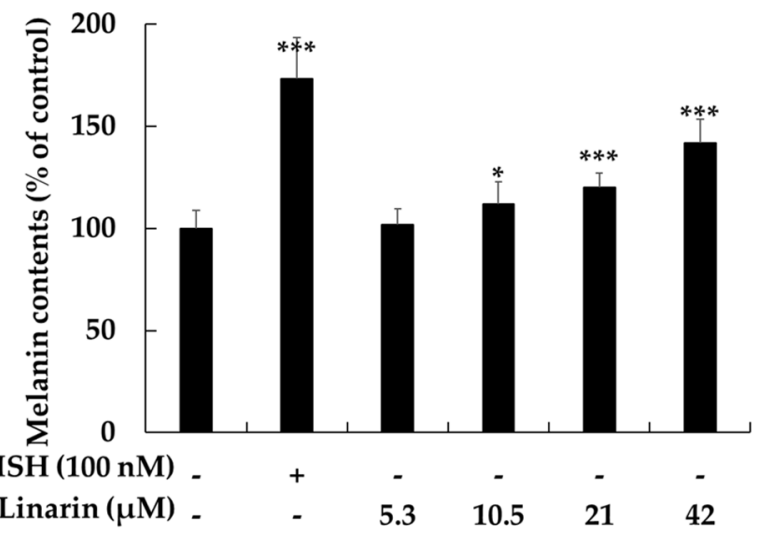

(C)

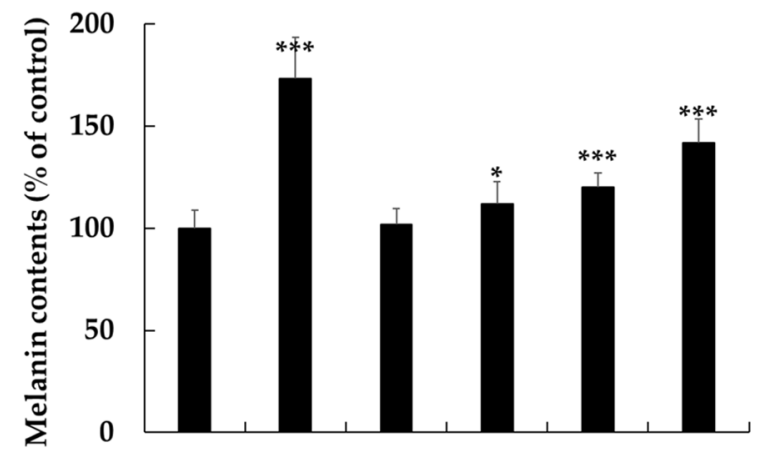

$\alpha$-MSH (100 nM)

Linarin $(\mu \mathrm{M})$
5.3
10. $\overline{42}$

Fig. 1 Effect of linarin on the viability and melanogenesis of B16F10 cells. The cells were treated with linarin $(5.3-84 \mu \mathrm{M})$ and alphamelanocyte stimulating hormone $(\alpha-\mathrm{MSH})(100 \mathrm{nM})$ for $72 \mathrm{~h}$. The cell viability (A), melanin content (B), and tyrosinase activity (C) have been expressed in percentages relative to non-treated cells. The $\alpha$-MSHtreated cells were used as the positive control. Data are presented as mean \pm standard deviation of three independent experiments $(n=3)$. ${ }^{*} p<0.05,{ }^{* *} p<0.01$ and ${ }^{* * *} p<0.01$ vs. non treated control

$\mu \mathrm{M}$. Cell viability was more than $87.7 \%$ even with the highest concentration of $84.0 \mu \mathrm{M}$. Therefore, we concluded that linarin is not toxic to B16F10 cells at concentrations less than $42.0 \mu \mathrm{M}$. Subsequent experiments were performed with linarin at concentrations of $5.25,10.5,21.0$, and $42.0 \mu \mathrm{M}$ that did not show cytotoxicity.

Next, we determined the effect of linarin on melanin biosynthesis. Melanin content was measured after incubating the cells with linarin $(5.3,10.5,21.0$, and $42.0 \mu \mathrm{M})$ for $72 \mathrm{~h}$. Alphamelanocyte stimulating hormone, a major factor that stimulates melanin biosynthesis in the skin, was used as the positive control [8]. As shown in Fig. 1B, when the melanin content in the untreated control group was $100 \%$, the melanin content in the positive control group was $173.2 \%$. In the test group, melanin content was 101.7, 111.8, 119.9, and $141.7 \%$ at linarin concentrations $5.3,10.5,21.0$, and $42.0 \mu \mathrm{M}$, respectively. These results showed that melanin levels were significantly increased by linarin in a concentration-dependent manner, indicating that linarin stimulates melanogenesis in B16F10 cells with no cytotoxicity. Tyrosinase is a rate-limiting enzyme involved in the oxidation of tyrosine and 3,4-dihydroxy-l-phenylalanine (L-dopa) in the initial stage of melanin synthesis [9]. Tyrosinase activity was subsequently analyzed to elucidate the underlying mechanisms of a linarin-induced enhancement in melanin synthesis in B16F10 cells. After incubating the B16F10 cells with different concentrations of linarin $(5.3,10.5,21.0$, and $42.0 \mu \mathrm{M})$ for $72 \mathrm{~h}$, the intracellular tyrosinase activity was measured. As shown in Fig. 1C, linarin significantly activated the intracellular tyrosinase activity in a concentration-dependent manner. Compared with the untreated control group, the tyrosinase activity of the positive control group increased by $206.6 \%$, whereas that of the linarin treated group $(5.3,10.5,21.0$, and $42.0 \mu \mathrm{M})$ increased by 98.0 , $100.6,117.9$, and $133.7 \%$, respectively.

Furthermore, tyrosinase level in B16F10 cells was determined by western blotting. At $72 \mathrm{~h}$ after linarin treatment, the tyrosinase level was significantly increased in a concentration-dependent manner. TRP-1, along with tyrosinase, regulates melanin synthesis. Therefore, we also examined TRP-1 level by western blotting. As shown in Fig. 2A, TRP-1 level was increased by linarin treatment. In particular, at $42.0 \mu \mathrm{M}$, the levels of tyrosinase $(318.6 \%)$ and TRP-1 (152.6\%) were increased by approximately $75 \%$ compared with the untreated control. These results indicated that linarin enhances melanogenesis via the regulation of melanogenic protein expression.

MITF is the master regulator of melanogenesis that upregulates melanogenic genes (tyrosinase, TRP-1, and TRP-2) by binding to the M-box motif in their promoter regions [10]. Thus, we further assessed MITF level after linarin treatment. As shown in Fig. 2B, 


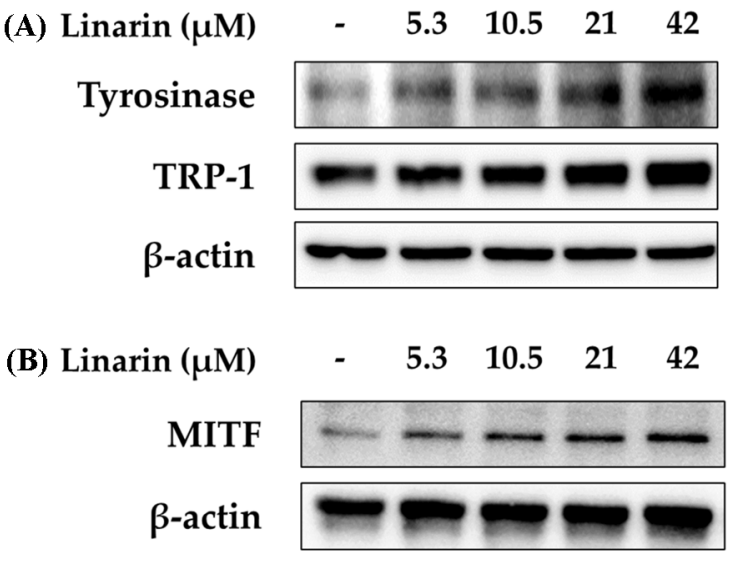

(C) Linarin $(\mu \mathrm{M})$
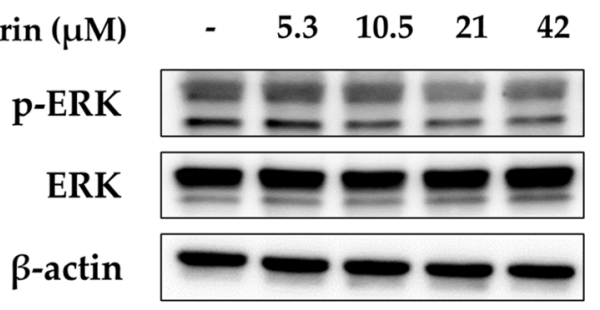

(D) Linarin $(\mu \mathrm{M})$

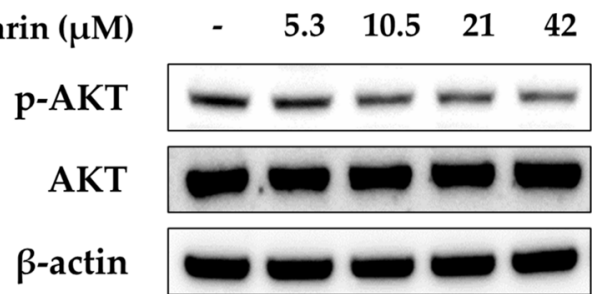

p-AKT
AKT
$\beta$-actin

\section{2}

Fig. 2 Effect of linarin on the expression of tyrosinase-related protein 1 (TRP-1), tyrosinase microphthalmia-associated transcription factor (MITF), pextracellular signal-regulated kinase (ERK), and protein kinase B (p-AKT) in B16F10 cells. The cells were treated with linarin $(5.25,10.5,21$, and $42 \mu \mathrm{M}$ ) for $48 \mathrm{~h}$. Western blotting was performed to determine the protein levels of TRP-1/tyrosinase (A), MITF (B), p-ERK (C), and p-AKT (D). $\beta$ actin was used as the loading control. TRP-1/tyrosinase, MITF, p-ERK, p-AKT, and $\beta$-actin were quantified using Image J (E-H)

the MITF level in B16F10 cells was noticeably induced after linarin treatment in a concentration-dependent manner. In particular, at $42.0 \mu \mathrm{M}$, the MITF level was approximately $163.2 \%$ higher than that of the untreated control group. These results suggested that MITF is involved in the linarin-induced activation of melanogenesis.

Previous studies [11] have reported that ERK and phosphatidylinositol 3-kinase/protein kinase B signaling pathways negatively regulate the melanogenesis in melanocytes and melanoma cells. Therefore, we investigated whether linarin affects these signaling pathways in $\mathrm{B} 16 \mathrm{~F} 10$ cells. Western blotting was performed to evaluate the effect of linarin on ERK and AKT phosphorylation. As shown in Fig. 2C and 2D, linarin treatment decreased the phosphorylation of ERK and AKT proteins. Linarin decreased the phosphorylation of ERK and AKT by 28.1 and $43.7 \%$, respectively, at a concentration of $42.0 \mu \mathrm{M}$ compared with the control group. These results suggested that linarin regulates ERK and PI3K/AKT signaling pathways in $\mathrm{B} 16 \mathrm{~F} 10$ cells, and might be responsible for upregulating melanogenesis.

Taken together, these results showed that linarin increases melanogenesis by upregulating the MITF gene, a critical gene associated with the expression of melanogenic genes such as tyrosinase and TRP. In addition, the linarin-induced upregulation of MITF was dependent on the inhibition of ERK and AKT signaling pathways. These results suggested that linarin can be further explored for its potential use in the treatment of hypopigmentation disorders such as albinism and vitiligo.

Acknowledgments This research was supported by the Ministry of Trade, Industry \& Energy (MOTIE), Korea Institute for Advancement of Technology (KIAT) through the Industry Innovation Foundation Project (P0018139).

\section{References}

1. Qu Y, Zhan Q, Du S, Ding Y, Fang B, Du W, Wu Q, Yu H, Li L, Huang W (2020) Catalysis-based specific detection and inhibition of tyrosinase and their application. J Pharm Anal 10: 414-425. doi: 10.1016/j.jpha. 2020.07.004.

2. Agarwal K, Podder I, Kassir M, Vojvodic A, Schwartz RA, Wollina U, Valle Y, Lotti T, Rokni GR, Grabbe S, Goldust M (2020) Therapeutic options in vitiligo with special emphasis on immunomodulators: A comprehensive update with review of literature. Dermatol Ther 33: e13215. doi: 10.1111/dth.13215

3. Lv J, An X, Jiang S, Yang Y, Song G, Gao R (2020) Protoporphyrin IX Stimulates Melanogenesis, melanocyte dendricity, and melanosome transport through the cGMP/PKG pathway. Front Pharmacol 11: 569368. doi: 10.3389/fphar.2020.569368

4. Qi W, Chen Y, Sun S, Xu X, Zhan J, Yan Z, Shang P, Pan X, Liu H (2021) Inhibiting TLR4 signaling by linarin for preventing inflammatory response in osteoarthritis. Aging (Albany NY) 13: 5369-5382. doi: 10.18632/aging.202469

5. Pan H, Zhang J, Wang Y, Cui K, Cao Y, Wang L, Wu Y (2019) Linarin improves the dyskinesia recovery in Alzheimer's disease zebrafish by inhibiting the acetylcholinesterase activity. Life Sci 222: 112-116. doi: 10.1016/j.lfs.2019.02.046

6. Nugroho A, Lim SC, Choi J, Park HJ (2013) Identification and quantification of the sedative and anticonvulsant flavone glycoside from Chrysanthemum boreale. Arch Pharm Res 36: 51-60. doi: 10.1007/ s12272-013-0015-8

7. Fernández S, Wasowski C, Paladini AC, Marder M (2004) Sedative and sleep-enhancing properties of linarin, a flavonoid-isolated from Valeriana officinalis. Pharmacol Biochem Behav 77: 399-404. doi: 10.1016/j.pbb. 2003.12.003

8. Fu T, Chai B, Shi Y, Dang Y, Ye X (2019) Fargesin inhibits melanin synthesis in murine malignant and immortalized melanocytes by regulating PKA/CREB and P38/MAPK signaling pathways. J Dermatol Sci 94: 213-219. doi: 10.1016/j.jdermsci.2019.03.004

9. Hwang YS, Oh SW, Park SH, Lee J, Yoo JA, Kwon K, Park SJ, Kim J, Yu E, Cho JY, Lee J (2019) Melanogenic effects of maclurin are 
mediated through the activation of cAMP/PKA/CREB and p38 MAPK/ CREB signaling pathways. Oxid Med Cell Longev 2019: 9827519. doi: $10.1155 / 2019 / 9827519$

10. Ko JH, Lee N, Kang HK, Kim BS, Lee JN, Hyun CG (2020) Antimelanogenic effects of hot-water extracts from Torreya nucifera via MAPKs and cAMP signaling pathway on B16F10 cells. Pharmazie 75:
565-570. doi: 10.1691/ph.2020.0522

11. Sangkaew O, Yompakdee C (2020). Fermented unpolished black rice (Oryza sativa L.) inhibits melanogenesis via ERK, p38, and AKT phosphorylation in B16F10 melanoma cells. J Microbiol Biotechnol 30: 1184-1194. doi: 10.4014/jmb.2003.03019 